\title{
VALORACIÓN ÉTICA DEL DIAGNÓSTICO DE ENFERMEDADES FUNDANTES DE DISCAPACIDAD EN LA VIDA PRENATAL
}

\begin{abstract}
Graciela Moya ${ }^{1}$
Resumen: La finalidad del diagnóstico prenatal genera un dilema controversial. Algunos autores sostienen que favorece el mejor desarrollo y adaptación del niño por nacer y sus familias, porque, al definir el estatuto moral del feto como inherente, debe recibir las mismas consideraciones morales que el niño ya nacido. Otros consideran que son medidas preventivas que disminuyen la prevalencia de enfermedades genéticas, al evitar el nacimiento de nińos con estas patologías. Así, el nińo por nacer no es considerado como persona y la discapacidad se interpreta como un daño para el niño y su familia, y debe ser evitada. Hay tensión al determinar si el diagnóstico prenatal es una práctica médica al servicio del hombre, con el objetivo de limitar el daño y sufrimiento humano, o bien una práctica eugenésica liberal. Tres conceptos se involucran en este conflicto: el de persona, el de discapacidad y el de prevención de enfermedades. El objetivo del artículo es reflexionar acerca de la interpretación de estos conceptos en diferentes visiones bioéticas, para alcanzar una mejor compresión de los mismos y aplicar los avances tecnológicos respetando la condición humana.
\end{abstract}

Palabras clave: diagnóstico prenatal, discapacidad, persona, prevención primaria, eugenesia

\section{Ethical evaluation of the diagnosis of diseases causing disability in prenatal life}

\begin{abstract}
The goal of prenatal diagnosis causes a controversial dilemma. Some authors sustain that it favors better development and adaptation of the child to be born and family, since, by defining the moral statute of the fetus as inherent, he/she must receive the same moral considerations that the child already born. Others consider that avoiding the birth of children with these pathologies is a preventive measure to diminish the prevalence of genetic diseases. Thus, the child to be born is not considered a person and the disability is interpreted as damage for the child and his/her family, which must be avoided. There is tension to determine whether prenatal diagnosis is a medical practice to the service of human beings with the goal to limit damage and human suffering, or rather an eugenic liberal practice. Three concepts are involved in this conflict: the person, disability and prevention of diseases. The goal of this article is to reflect about the interpretation of these concepts in different bioethical views, in order to reach a better understanding of them and applying technological advances while respecting human condition.
\end{abstract}

Key words: prenatal diagnosis, disability, personhood, primary prevention, eugenic practice

\section{Valoraçáo ética do diagnóstico de enfermidades causadoras de descapacidade na vida pré-natal}

Resumo: A finalidade do diagnóstico pré-natal gera um dilema controvertido. Alguns autores sustentam que favorece o melhor desenvolvimento e a adaptação da criança por nascer e suas famílias, porque, ao definir o estatuto moral do feto como inerente, deve receber as mesmas consideraçóes morais que a criança nascida. Outros consideram que são medidas preventivas que diminuem a prevalência de enfermidades genéticas, ao evitar o nascimento de crianças com estas patologias. Assim, a criança por nascer não é considerada como pessoa e a descapacidade se interpreta como um dano para a criança e sua família, e deve ser evitada. Há tensão ao determinar se o diagnóstico pré-natal é uma prática médica a serviço do homem, com o objetivo de limitar o dano e sofrimento humano, ou uma prática eugênica liberal. Três conceitos são envolvidos neste conflito: o de pessoa, o de descapacidade e o de prevenção de enfermidades. O objetivo do artigo é refletir acerca da interpretação destes conceitos em diferentes visóes bioéticas, para alcançar uma melhor compreensáo deles e aplicar os avanços tecnológicos respeitando a condição humana.

Palavras-chave: diagnóstico pré-natal, descapacidade, pessoa, prevenção primária, eugenia

\footnotetext{
${ }^{1}$ Instituto de Bioética, Facultad de Ciencias Médicas, Pontificia Universidad Católica Argentina, Argentina Correspondencia: gracielamoya@uca.edu.ar
} 


\section{Introducción}

Los avances en el conocimiento en genética médica conllevan la promesa de desarrollar tratamientos y curas para las enfermedades genéticas. Existe, no obstante, una gran discrepancia entre la capacidad diagnóstica y la posibilidad terapéutica, ya que, si bien es posible realizar diagnósticos muy certeros de enfermedades genéticas, desde etapas muy tempranas de la vida de la persona como en el estadio de embrión preimplantatorio o fetal-, estas no tienen un tratamiento curativo que evite su aparición o disminuya sus complicaciones clínicas. No obstante, los diagnósticos preimplantatorio y prenatal son considerados como una extensión lógica de los cuidados médicos rutinarios.

Muchos autores han remarcado el valor de estos diagnósticos por su efectividad y precocidad; sin embargo, su finalidad genera un dilema controversial. Por un lado, algunos autores sostienen que estos diagnósticos tempranos permiten establecer medidas terapéuticas y contención familiar necesaria —en los niveles emocional y social—, que facilitan el mejor desarrollo y adaptación del niño por nacer y sus familias. Así, definen el estatuto moral del embrión y del feto como inherente, por lo cual deben recibir las mismas consideraciones morales que el niño ya nacido respecto de su derecho a la vida, cuidado, y protección. Otros, empero, consideran que estos diagnósticos son medidas preventivas para disminuir la prevalencia de enfermedades genéticas, especialmente en las familias de alto riesgo, al evitar la transferencia de embriones o el nacimiento de niños con estas patologías. Entonces, consideran que existe una obligación moral hacia el hijo y aún hacia la sociedad de tener el mejor hijo posible. El niño por nacer no es considerado como persona y la discapacidad asociada a estas enfermedades se interpreta como un daño para la madre, el niño y su familia, y para la sociedad, que debe ser evitado.

Aquí surge un intenso punto de tensión y división de corrientes de pensamientos respecto de los diagnósticos tempranos de patologías que producen discapacidad en la vida postnatal. El dilema se centra en determinar si es una práctica médica al servicio del hombre, con el objetivo de limitar el daño y sufrimiento de las personas con des- ordenes genéticos, y mantener el respeto por la integridad y dignidad de la persona humana, o bien una práctica eugenésica liberal que, al evitar el nacimiento de personas con enfermedades genéticas, disminuye la prevalencia de la discapacidad, pero sostiene la discriminación hacia las personas con enfermedades fundantes de discapacidad. Tres conceptos se involucran en este conflicto, el de persona humana y cómo se aplica el mismo en la etapa prenatal de la vida del ser humano; el de discapacidad, interpretado desde diferentes perspectivas, y el de prevención de enfermedades genéticas, desde la práctica clínica y la salud pública.

La interpretación de las diferentes definiciones antropológicas, teorías éticas y perspectivas respecto de la persona, la enfermedad y la discapacidad pueden derivar en conclusiones muy diferentes o explicar cursos de acción contradictorios. El objetivo del trabajo es realizar una reflexión acerca de la interpretación de estos conceptos en diferentes visiones bioéticas, que facilite una más acabada compresión de los mismos y permita aplicar los avances tecnológicos de manera que respeten la condición humana, al aceptar su diferencia, protegerla en su vulnerabilidad y facilitar su bienestar.

\section{Conceptos para la discusión}

1. Interpretación del concepto de persona en la etapa prenatal de la vida del ser humano.

Definir cómo se aplica el concepto de persona en las etapas tempranas —embrionaria o fetal- de la vida prenatal del ser humano es un tema que puede ser esclarecido desde una visión transdisciplinaria, que involucre a las ciencias biológicas, la filosofía, la teología, la ética y el Derecho. Tanto en los aspectos filosóficos y éticos como teológicos existen variadas corrientes de pensamientos e interpretaciones que generan dilemas en la definición del concepto de persona. Desde el punto de vista legal, cada sociedad determina qué valores deben considerase de justo respeto, y requerir una regulación y protección especial. Sin embargo, desde la perspectiva biológica, al tratarse de una ciencia de primer grado de abstracción, las evidencias son objetivas y demostrables, y basadas en el método científico. Desde el momento de unión 
de las membranas del óvulo y espermatozoide se desencadenan procesos biológicos en un orden gradual, continuo y coordinado de desarrollo. Este proceso de desarrollo es intrínseco del embrión - ya que depende de la organización de su genoma-, pero, a su vez, se sostiene en la íntima relación existente entre el embrión y el endometrio materno en los momentos previos y durante su implantación(1).

Toda esta información científica hace que se vuelva irrelevante cuestionarse cuándo comienza una nueva vida humana. Actualmente, la cuestión es definir el instante a partir del cual la vida humana adquiere significado moral, y, en consecuencia, cómo deben ser respetados moralmente los embriones y fetos humanos. Ciccone(2) propone evaluarlo, con un orden lógico, desde tres aspectos:

- Estatuto ontológico: quién o qué es el.

- Estatuto ético: qué deberes se tiene respecto del ser humano en la vida prenatal.

- Estatuto jurídico: cuáles de estos deberes es necesario legislar y reglamentar y qué derechos se deben reconocer y tutelar al ser humano en la vida prenatal.

\subsection{Estatuto ontológico.}

Visión sustancialista: Esta perspectiva equipara como sinónimos los conceptos de "persona" y "ser humano", ya que define a la persona como un ser sustancial de naturaleza racional, basada en la definición clásica de "persona" establecida por Boecio(2). Esta naturaleza racional es intrínseca a su ser, por ello se mantiene durante toda su existencia desde las etapas iniciales de su vida hasta su muerte natural. Tiene una sustancia interna natural cuya unidad estructural está ordenada al desarrollo de sus facultades fundamentales, que se manifiestan en una serie de capacidades, actividades y funciones que, sin duda, pueden ser consideradas como características de la racionalidad, pero no reducidas a ellas. Un ser sustancial no puede cambiar sus capacidades fundamentales, es decir, no puede perder su naturaleza fundamental y continuar su existencia(3). La persona humana es un organismo vivo, cuya característica diferen- cial respecto de otros seres es su facultad de ser un agente moral racional. Esta facultad se mantiene idéntica durante su existencia, aun cuando no exprese las funciones, conductas, o actividades que típicamente se atribuyen a los agentes morales racionales, activos y maduros. Al ser un agente moral racional, tiene un valor moral intrínseco mientras dure su existencia(4), porque es propio de su naturaleza. Por lo tanto, desde las etapas muy tempranas de la vida del ser humano - embrionaria y fetal- es el mismo individuo concreto, dotado de naturaleza ontológica, ya que los seres sustanciales son ontológicamente primero que sus partes o capacidades(4). El individuo y sus partes, y su rol en la actualización de las capacidades básicas, tienen una finalidad intrínseca porque su objetivo es mantener la unidad del individuo y su perfección como un todo. El individuo puede adquirir o perder parte de o todas sus capacidades, pero mantiene su naturaleza humana idéntica en el tiempo. Esto implica que posee un dignidad intrínseca, por lo tanto, su derecho a la vida se sostiene en su naturaleza, no es adquirido por sus capacidades u otorgado por terceros.

En resumen, si se define al ser humano en la vida prenatal como ser individual, concreto y único, considerar una humanización indirecta o gradual es difícil de sostener. El ser humano "es" persona en virtud de su naturaleza racional, no se "convierte en" persona debido al efectivo ejercicio de determinadas funciones (como son la capacidad de relacionarse, la sensibilidad o la racionalidad). El ser persona pertenece, entonces, al orden ontológico, no se puede adquirir ni disminuir gradualmente, independientemente de su estadio de desarrollo físico o social(6).

Visión empirista of funcionalista: Esta perspectiva considera que hay diferencia entre los conceptos "persona" y "ser humano" y utilizar estos términos como sinónimos lleva a confusión(7). Por ello, definen "persona" como cualquier ser, humano u otro, que tiene la función mental suficiente para que se considere su destrucción deliberada como intrínsecamente mala. Mientras que el término "ser humano" se refiere a cualquier ser miembro de la especie Homo Sapiens, sin considerar la naturaleza de su vida mental(7). Del mismo modo, John Locke (1690)(8) también define el concepto de persona principalmente por sus funciones 
racionales y su autoconciencia: "un ser pensante inteligente, provisto de razón y de reflexión, y que puede considerarse a sí mismo como sí mismo, una misma cosa pensante en diferentes tiempos y lugares; lo que tan solo hace porque tiene conciencia, que es inseparable del pensamiento". Engelhardt(9) también propone una distinción entre persona y ser humano a partir de los criterios de actualidad: "no todos los seres humanos son personas, no todos son autoreflexivos, racionales o capaces de formarse un concepto sobre el mérito de la culpa o del elogio. Los fetos, las criaturas, los retrasados mentales profundos y los que se encuentran en coma profundo son ejemplos de seres humanos que no son personas". Sostiene así el concepto de persona en la expresión de las capacidades naturales humanas y no sólo en su naturaleza humana. Estas capacidades pueden establecerse en grados — por ejemplo, racionalidad, autoconciencia, habilidad para comunicarse, entre otras. Es decir, ser persona se puede interpretar como una propiedad mensurable, y sólo aquellos seres humanos que cumplan con un determinado umbral de capacidades pueden ser considerados personas. Entonces, es necesario diferenciar dos conceptos: las facultades naturales que son propias de cada ser, y las habilidades ejecutables por cada persona. Las facultades naturales no vienen en grados porque dependen de la naturaleza del ser, pero las habilidades sí son mensurables(4). Si el ser persona y su dignidad vienen en grados, no todos los seres humanos tienen el mismo valor moral intrínseco, sino que este será un atributo adquirido o perdido en forma cuantitativa. En ese caso, el respeto por la dignidad de la persona humana, y en consecuencia su derecho a la vida, serán graduales y se aplicarán en ciertas etapas de su vida y no en otras.

\subsection{Estatuto moral.}

La definición de estatuto moral es controversial. Se puede interpretar como una característica propia del ser, que deviene de su dignidad ontológica, una propiedad intrínseca de la naturaleza humana y de la que emanan los derechos atribuibles a todos los seres humanos(5). O bien, como una característica cuantitativa adquirida u otorgada. Significa ser moralmente considerado por otros, tener una posición moral o ser protegido por normas morales, incluyendo principios, re- glas, obligaciones y derechos(10). En estas perspectivas, implica ser una entidad hacia la cual los agentes morales pueden tener obligaciones morales; es decir, considerar sus necesidades, intereses o bienestar, en las deliberaciones acerca de esa en$\operatorname{tidad}(11)$.

En este caso, la controversia consiste en definir los criterios que deben ser evaluados para adscribir el estatuto moral a diversos tipos de entidades. Miller(12) sostiene que una entidad puede ser un agente moral cuando es capaz de una acción que puede ser evaluada como correcta o incorrecta; o un paciente moral, el trato que recibe puede ser evaluado como correcto o incorrecto. Serían agentes morales las personas adultas normales, probablemente los animales adultos de las especies superiores, o quizá las corporaciones y las naciones, pero no las personas muy jóvenes, los insanos, los severamente retardados o los comatosos. Un niño pequeño, por ejemplo, no sería un agente moral, pero sí un paciente moral, ya que es importante valorar la manera en cómo es tratado. Engelhardt(9), también considera que no todos los seres humanos son iguales; sólo las personas y no los seres humanos pueden ser incluidos en las discusiones éticas, por ser especiales. Surge el concepto de que un ser humano comienza su existencia sin ser persona, luego se desarrolla como persona y después en alguna etapa de su vida puede dejar de ser persona, aunque continua siendo ser humano.

Harris(13) propone un flujo continuo de vida humana que comienza con los gametos y continúa con un individuo. El ser humano transita su vida en etapas que designa: "pre-personas" - "personas" - "ex-personas". El criterio que define la aparición o desaparición de la persona es la capacidad de valorar su propia existencia. Esta visión genera dificultades filosóficas, especialmente relacionadas con la idea de la identidad(14). Se justifica por la negación de la persona como ser complejo, jerárquico e integrado(15). Así, se construye un concepto de persona humana, no objetivo u ontológico, sino basado en propósitos subjetivos o intersubjetivos en función de sus capacidades o en función de su utilidad. El concepto de persona es considerado como un "constructo social", es decir, como un atributo conferido socialmente a algún ser humano por otros seres humanos(14). 
Por lo tanto, el concepto de persona y de su estatuto moral en etapas tempranas del desarrollo de la vida puede interpretarse desde distintas corrientes de pensamiento $y$, por tanto, otorgarse un distinto respeto por la vida de los seres humanos por nacer.

2. El concepto de discapacidad interpretado desde diferentes perspectivas.

En los últimos años se introduce el cuestionamiento bioético en el concepto de discapacidad, ya que está imbuido por significados valorativos y descriptivos de la vida de las personas que la sufren(16). La Convención sobre los Derechos de las Personas con Discapacidad define: "Las personas con discapacidad incluirán a quienes tengan impedimentos físicos, mentales, intelectuales o sensoriales a largo plazo que, al interactuar con diversas barreras, pueden impedir su participación plena y efectiva en la sociedad, en igualdad de condiciones con los demás" (A/ AC.265/2006/4, anexo II) $(17,18)$. Este concepto tiene implicancias particulares en la vida prenatal, porque se predice la discapacidad de la persona con diagnóstico de alguna enfermedad genética. Entra en juego el concepto de que la discapacidad es constitutivo de la identidad de la persona(19), definiendo su estatus moral.

Dun(16) describe que habría cinco maneras de "pensar y hablar sobre discapacidad", dependiendo de dónde se pongan los aspectos valorativos y descriptivos, y cómo se consideren los imperativos morales: el modelo médico, el modelo social, el modelo fenomenológico, el modelo bienestarista y el modelo de las capacidades. Los dos primeros ocupan dos extremos, definiendo discapacidad en términos médicos e individuales, o en términos sociales. A partir de estos extremos surgen nuevas perspectivas en las que intervienen una combinación de ambos aspectos. Concluye que estos modelos pueden dar soporte a la ética clínica, tanto en la toma de decisiones como en el marco del proceso de sostén de la bioética.

2.1. Modelo médico: Considera que la discapacidad es una dificultad física o psicosocial, entendida como una desviación estadística del promedio normal de funcionamiento normal del ser humano(18). Por lo tanto, existiría algo intrínseca- mente malo en tener una discapacidad, porque la persona funcionaría por debajo del rango normal, haciendo su vida menos buena y disminuyendo su calidad de vida. Implica una descripción negativa para la persona y se valora su vida en desventaja respecto de otras. La obligación moral es asistir a esta persona para lograr su "normalización”, removiendo lo que es intrínsecamente malo, orientado hacia el tratamiento médico en forma individual. En los casos en que no es posible remover la causa de la discapacidad, como las enfermedades genéticas, se considera el uso de las tecnologías reproductivas para evitar que una persona con estas enfermedades llegue a la existencia. Así, la normalización implica una obligación moral de que la persona se integre a una vida en un ambiente normal o bien generar un medio adecuado en instituciones con servicios especiales para aislarla del resto de la sociedad, y que reciba un apoyo extra que lo asista para este fin.

2.2. Modelo social: Se opone al modelo anterior. Sostiene que es no es válido realizar un juicio de valor basado en una desviación estadística del funcionamiento físico o psicosocial de una persona. No niega que las personas tienen dificultades en su desempeño físico o psicosocial, pero argumenta que es incorrecto decir que existe algo intrínsecamente negativo en el hecho de que algunas tengan ciertas capacidades y otras no, debido a sus patologías. El componente valorativo de este modelo se centra en el reclamo de que la sociedad ha fallado en adecuarse a la realidad de las diferencias individuales vinculadas con la discapacidad. La sociedad tiene la obligación moral de reconocer que la dificultad no es mala en sí misma, que debe encontrarse con las necesidades y modificar las condiciones ambientales y sociales en que se desarrollan las personas(18).

2.3. Modelo fenomenológico: Sitúa la experiencia individual de vivir con una cierta dificultad como centro del concepto de discapacidad(20). Incorpora el concepto médico, aceptando que se puede hacer una valoración acerca de la vida de las personas que tienen determinada dificultad que puede afectar su calidad de vida, pero también considera que tener una dificultad, aun severa, no debe considerarse como algo intrínsecamente malo, de manera que no exista una obligación moral de corregir esa dificultad. El alcance de la discapacidad 
de una persona depende enteramente de cómo ella experimenta la dificultad, tanto en su corporeidad como socialmente. Reconoce la discapacidad como contingente, existente en el aspecto físico de la persona, y que la experiencia de la misma está vinculada a la vivencia social, orientada relacionalmente, temporalmente contingente, y otorgando un marco de la experiencia individual de estar en el mundo(16). Entonces, la valoración ética será relativa a la experiencia de cada una de las personas y requerirá una visión que expanda el horizonte moral para entender y sostener mejor cada proyecto de vida individual(21).

2.4. Modelo bienestarista: El bienestar es el único valor que sostiene la moralidad y la maximización del bienestar debe ser la guía de la valoración mo$\mathrm{ral}(22)$. Es una teoría consecuencialista que sostiene que cualquier estado físico o psicosocial de la persona genera una discapacidad, si ese estado hace que la vida de esa persona sea probablemente peor en términos de su bienestar, en una determinada circunstancia social y ambiental. No considera la discapacidad como una desviación estadística de lo normal ni entiende la discapacidad en términos existencialistas; tampoco acepta que el daño asociado a la discapacidad es enteramente debido a un prejuicio o injusticia social, sino que lo considera como un estado generado por la interacción entre la biología o psicosociología de la persona y el ambiente que la rodea. Determinar si una persona tiene o no una discapacidad depende del juicio sobre el bienestar de esa persona. Observando quién es esa persona y cómo vive su vida, y si su vida implica un cierto bienestar, se evalúa sobre la base del bienestar de la persona y es relativo a la circunstancia. Por lo tanto, la obligación moral es maximizar el bienestar de la persona a través de dos caminos: cambiando sus circunstancias sociales y alterando sus propiedades físicas y psicosociales.

2.5. Modelo de las capacidades: La prosperidad de una persona depende de que tenga ciertas capacidades y oportunidades reales, basadas en sus circunstancias personales y sociales. Sin esas oportunidades, la vida puede verse empobrecida(23). La desigualdad de oportunidades permite que una persona con discapacidad tenga una desventaja en la sociedad. Por lo tanto, el imperativo moral es asegurar que reciba una igualdad de oportunida- des para la prosperidad. La medida en que las personas con discapacidad puedan desarrollarse con buena salud, actuar con base en sus pensamientos y emociones, desarrollar actividades personales y participar en actividades de la comunidad, políticas y económicas, determinará el ámbito de nuestro deber para ayudarlas a desarrollarse.

3. El concepto de prevención de enfermedades genéticas o anomalías congénitas desde la práctica clínica y la salud pública.

La mayoría de los problemas crónicos de salud en la infancia son debidos a enfermedades genéticas o malformaciones congénitas, y son causa de discapacidad motora, sensorial, visceral o intelectual. Las enfermedades genéticas y anomalías congénitas son desórdenes poco frecuentes en forma aislada, pero en su conjunto afectan a alrededor del $3-5 \%$ de los recién nacidos(24), son responsables de aproximadamente el $20-30 \%$ de las muertes infantiles(25) y del $30-50 \%$ de las muertes posneonatales(26). En Argentina representan la segunda causa de mortalidad infantil, siguiendo a las enfermedades perinatales(27).

En este contexto, los recientes avances en la tecnología en genética permitirán un más amplio interés médico y del público en general por utilizarlos para prevenir la discapacidad. Las estrategias de prevención, que incluyen las intervenciones de nivel primario, secundario y terciario, fueron propuestas hace más de 50 ańos y categorizan las intervenciones tanto médicas como de salud pública(28). Estos niveles de prevención en genética se aplican de la siguiente manera:

3.1. Prevención primaria: Busca evitar la ocurrencia de la enfermedad genética o anomalías congénitas. Para aquellas enfermedades poco frecuentes, génicas, o cromosómicas, las medidas de intervención médicas y comunitarias se centran en distintas estrategias de prevención:

a) Evitar la exposición a factores ambientales conocidos o sospechosos de ser teratogénicos.

b) Prevenir las enfermedades infecciosas, mediante la vacunación o evitar la exposición de mujeres gestantes. 
c) Incorporar nutrientes en la dieta, como el ácido fólico periconcepcional, que disminuye la ocurrencia y recurrencia de defectos del cierre del tubo neural.

d) Tratar enfermedades maternas, como la diabetes, la fenilcetonuria, o la hipertensión.

e) Detectar portadores de enfermedades genéticas y el asesoramiento genético preconcepcional puede facilitar la toma de decisiones reproductivas. En algunas comunidades existen programas de estudio de portadores, por ejemplo en la comunidad judía Ashkenazi en Estados Unidos(29)2.

3.2. Prevención secundaria: Una vez ocurrida la enfermedad, el objetivo se enfoca en evitar las manifestaciones clínicas, como con la detección temprana y la intervención en las fases preclínicas de la enfermedad. Un clásico ejemplo es el screening de recién nacido, para enfermedades como fenilcetonuria, galactosemia y la intervención temprana centrada en la dieta, o hipotiroidismo y la suplementación hormonal temprana. En estos casos se ejemplifica claramente la interacción entre los factores genéticos y ambientales en enfermedades con base principalmente genética, como la fenilcetonuria, en las que es manifiesto el impacto de una dieta pobre en fenilalanina en la expresión de la enfermedad.

\subsection{Prevención terciaria: Se centra en intentar re-} ducir los efectos de la enfermedad diagnosticada y las manifestaciones clínicas evidentes por la prevención de las complicaciones, secuelas y deterioro. En estos casos, las actividades están centradas en los tratamientos médicos, quirúrgicos, y de rehabilitación e integración a la sociedad. Uno de los desafíos más importantes en las enfermedades genéticas es que muchas de las condiciones que causan discapacidad intelectual no pueden ser revertidas una vez que ocurre el déficit intelectual. Esto implica que es necesaria la intervención y cooperación de varios especialistas, y que eventualmente deben implementarse medidas previas al nacimiento.

\footnotetext{
${ }^{2}$ Dor Yeshorim: sistema de detección genética confidencial e internacional usado por judíos ortodoxos. Intenta prevenir la transmisión de desórdenes genéticos que tienen una mayor prevalencia en las comunidades judías ashkenazíes. El sistema fue establecido para seguir la ley judía, que no permite el aborto, pero evita la concepción de niños con estos desordenes al analizar a jóvenes adultos antes de casarse.
}

\section{Discusión}

Si bien la aplicación de estos términos es clara en la prevención de enfermedades en general, es posible que se origine confusión cuando se relaciona con la prevención de las enfermedades genéticas y anomalías congénitas, ya que algunos autores incluyen en el concepto de prevención primaria el diagnóstico prenatal y la interrupción voluntaria del embarazo, y el diagnóstico de preimplantación y la selección embrionaria. El conflicto surge porque en este caso no se evita la ocurrencia de la enfermedad sino que se evita el nacimiento del niño o la transferencia de embriones portadores de esa enfermedad. Esto se sostiene con una perspectiva de que la discapacidad es una tragedia individual y familiar, y una catástrofe social, y la aceptación más amplia del aborto selectivo de personas con enfermedades que generen discapacidad, considerando en forma extrema al aborto del niño por nacer como tratamiento médico. Actualmente existe la posibilidad técnica de elegir las características genéticas que nuestros hijos porten, no por la manipulación de su genoma, sino por la selección y descarte entre un rango de embriones o fetos. Por ello, algunos autores sostienen que el diagnóstico prenatal discrimina en contra de las personas con discapacidad y coloca a las mujeres en una situación de vulnerabilidad, ya que muchas veces se interpreta que son responsables de la patología del niño por no haber realizado los estudios diagnósticos en tiempo de detectarla tempranamente. En estos casos, la actitud de los médicos y del sistema de salud es condicionante de las decisiones maternas y del respeto de la vida del niño por nacer.

Este conflicto surge particularmente en aquellas enfermedades genéticas que actualmente no tienen un tratamiento curativo, reconociéndose un vínculo estrecho entre genética y discapacidad intelectual, en el que prevalece la perspectiva del modelo médico del concepto de discapacidad. Esto puede generar que no se consideren otros modelos, como aquellos que se centran en las necesidades de las personas o en la problemática social, y así se corre el riesgo de que se disminuya la importancia y la motivación de los investigadores hacia el tratamiento y curación de los desórdenes genéticos, ya sea in utero o luego del nacimiento del niño, o hacia la búsqueda de estrategias que 
faciliten la integración social de las personas con enfermedades genéticas que se vinculen a discapacidad en la vida postnatal.

Surge el dilema acerca de la finalidad del diagnóstico prenatal: si realmente beneficia directamente al feto, permitiendo un diagnóstico e intervención temprana, o si su propósito es facilitar la elección reproductiva de las mujeres y de su familia, y así seleccionar el tipo de hijo que se desea tener. En esta visión, Parens y Asch(30) han articulado, desde los derechos de las personas con discapacidad, una crítica del diagnóstico prenatal considerando los siguientes tres puntos:

1. El diagnóstico prenatal, en lugar de mejorar la situación médica y social de las personas con discapacidad hoy o en el futuro, refuerza la visión del modelo médico, esto es, que la discapacidad en sí misma y no la discriminación hacia las personas con discapacidad es el problema que debe ser resuelto.

2. Al evitar el nacimiento de un niño por su posible discapacidad, los padres sugieren que ellos no desean aceptar un niño diferente al que esperan, y una característica del niño, como una patología física o intelectual, se convierte en la base del rechazo total del hijo, sin considerar otras características.

3. Cuando los futuros padres seleccionan en contra de un feto por su posible discapacidad, realizan una elección desafortunada, muchas veces poco informada, pensando que el niño no tendrá la calidad de vida que ellos buscan para sus hijos.

Los autores refieren en estos tres puntos que el diagnóstico prenatal seguido del aborto es moralmente problemático y está dirigido habitualmente por la desinformación, ya que existe una enorme brecha entre el conocimiento teórico de la enfermedad genética y el conocimiento de la vida de las personas con esa enfermedad. Esto genera una caracterización y estigmatización injustas, que pueden expresar mensajes negativos acerca de su valor y reforzar su exclusión y desventaja social. Si los padres reciben acerca de la potencialidad de desarrollo de sus hijos una información limitada o incorrecta, será imposible que adopten una de- cisión informada acerca de los mejores controles pre, peri y posnatales necesarios.

Desear tener un hijo sano no implica que, si el niño no lo es, no será amado o cuidado cuando se diagnostique la enfermedad en la vida posnatal, o que los padres no le darán el sostén, amor y cuidado que requiera. El desafío es encontrar un camino que permita ubicar los métodos diagnósticos prenatales de manera que honren la dignidad de la persona humana y permitan orientar el valor de las intervenciones en forma evidente hacia la persona en gestación.

\section{Conclusiones}

Existen diferentes interpretaciones del concepto de persona en la vida prenatal, ya sea se defina este desde una perspectiva ontológica o sustancialista, o desde una visión empirista o fenomenológica. También, diferentes visiones del concepto de "discapacidad", según se centre en los aspectos médicos, sociales o bien en la persona misma. Aunque se considere la finalidad de disminuir la prevalencia de enfermedades que fundan la discapacidad como un bien para la persona, en forma individual, para la familia y para la sociedad en su conjunto, este concepto debe diferenciarse claramente del concepto eugenésico de evitar el nacimiento de las personas diagnosticadas con estas patologías en las etapas tempranas de su vida. El derecho a la vida de todos los seres humanos se interpreta como inherente, no como un derecho adquirido u otorgado por otros; por ello, se deben adoptar todas las medidas para garantizarlo a todas las personas. Es un desafío establecer políticas públicas que promuevan eficientemente el avance del desarrollo científico y, simultáneamente, la contención e inclusión de las personas con enfermedades que generen discapacidad en la sociedad. La propuesta es la implementación de educación académica y pública acerca de la visión de las personas con enfermedades que generen discapacidad, y de la definición del valor que tiene la vida humana en nuestra sociedad. Es una tarea que involucra a los pacientes y sus familias, a los médicos, a las organizaciones científicas y gubernamentales y al público en general. El Comité de los Derechos del Niño, dependiente de Naciones Unidas, publicó en 2006 la Observación General Número 9 acerca de los derechos de los nińos con 
discapacidad. En el artículo 6, sobre el derecho a la vida, la supervivencia y el desarrollo de los niños con discapacidad, hace un llamado contra el infanticidio y el aborto e "insta a los Estados Partes a que adopten todas las medidas necesarias para poner fin a esas prácticas, en particular aumentando la conciencia pública, estableciendo una legislación apropiada y aplicando leyes que garanticen un castigo adecuado a las personas que directa o indirectamente violan el derecho a la vida, la supervivencia y el desarrollo de los niños con discapacidad"(31).

\section{Referencias}

1. Edwards A. Aspects of the molecular regulation of early mammalian development. Reproductive BioMedicine Online 2003; 6(1): 97-113.

2. Ciccone L. Bioética: Historia, Principios, Cuestiones. Madrid: Editorial Palabra; 2005.

3. Moreland JP. Humanness, personhood, and the right to die. Faith and Philosophy 1995; 12(1): 95-112.

4. Beckwith FJ. The Human Being, a Person of Substance: A Response to Dean Stretton. In: Napier S, (eds.) Persons, Moral Worth, and Embryos, Philosophy and Medicine. New York: Springer Science+Business Media B.V.; 2011: 67-83.

5. Eijek JW. Los criterios de la individualidad orgánica y el estatuto bioantropológico del embrión preimplantatorio. In Sgreccia E, Laffite J, (eds.) El embrión humano en la fase de preimplantación, aspectos cientificos y consideraciones bioéticas. Madrid: Biblioteca de Autores Cristianos; 2008.

6. Palazzani L. I significati filosofici del concetto di persona. In Ciccone L. Bioética: Historia, Principios, Cuestiones, Madrid: Editorial Palabra; 2005.

7. Brown J. Research on human embryos - a justification. J Med Ethics 1986; 12(4): 201-206.

8. Locke J. An essay concerning human understanding. Berkely: University of California Press; 1975.

9. Engelhardt HT. The foundation of Bioethics. New York: Oxford University Press; 1996.

10. Beauchamp TL, Childress JF. Principles of Biomedical Ethics. New York: Oxford University Press; 2009.

11. Warren MA. Moral statute, obligations to persons and other living things. New York: Oxford University Press Inc.; 1997.

12. Miller HB. Science, Ethics, and Moral Status. Between the species 1994; 10: 10-18.

13. Harris J. The concept of the Person and the value of life. Kennedy Inst Ethics J. 1999; 9(4): 293-308.

14. Gillon R. To what do we have moral obligations and why? I. Br Med J (Clin Res Ed). 1985; 290(6482): 1646-1647.

15. Donadío Maggi de Gandolfi MC. La Nueva moral. Sapientia 1998; 53.

16. Dunn M. Discourse of disability and clinical ethics support. Clinical Ethic. 2011; 6: 32-38.

17. Oficina del Alto Comisionado de las Naciones Unidas para los Derechos Humanos. Convención sobre los derechos de las personas con discapacidad. [cited 2012 May 22] Available from: URL: http://www2.ohchr.org/spanish/law/disabilitiesconvention.htm

18. Palacios A. El modelo social de discapacidad: orígenes, caracterización y plasmación en la Convención Internacional sobre los Derechos de las Personas con Discapacidad. Madrid: Ed. Cinca; 2008.

19. Stainton T. Identity, difference and the ethical politics of prenatal testing. J Intellect Disabil Res. 2003; 47(Pt 7): 533539.

20. Scully JL. Disability bioethics: Moral bodies, Moral difference. Lanham MD: Bowman \& Littlefield; 2008.

21. Mackenzie C, Scully JL. Moral imagination, disability and embodiment. J APPL Philos. 2007; 24(4): 335-351.

22. Savulescu J, Kahane G. Disability: a welfarist approach. Clinical Ethics 2011; 6(1): 45-51.

23. Nussbaum M. Frontiers of justice: Disability, nationality, species membership. Cambridge, MA: Harvard University Press; 2006.

24. Robinson A, Linden MG. Clinical Genetic Handbook. Boston: Blackwell Scientific Publications; 1993.

25. Berry RJ, Buehler JW, Strauss LT. Birth weight-specific infant mortality due to congenital abnormalities, 1960 and 1980. Public Health Report 1987; 102(2): 171-181.

26. Hoekelman RA, Pless IB. Decline in mortality among young American during the $20^{\text {th }}$ century: Prospects for reaching national mortality reduction goals for 1990. Pediatrics. 1988; 82(4): 582-595.

27. Bronberg RA, Gutiérrez Redomero E, Alonso MC, Dipierri JE. Mortalidad infantil por malformaciones congénitas y condición socioeconómica: el caso de la Argentina. Rev Panam Salud Pública 2012; 31(6): 469-475.

28. Dolk H. What is the "primary" prevention of congenital anomalies? EUROCAT Project Management Committee. Lancet 2009; 374(9687): 378. 
Valoración ética del diagnóstico de enfermedades fundantes de discapacidad en la vida prenatal - Graciela Moya

29. Ekstein J, Katzenstein H. The Dor Yeshorim story: community-based carrier screening for Tay-Sachs disease. Adv Genet. 2001; 44: 297-310.

30. Parens E, Asch A. Prenatal testing and disability Rights. Washington DC: Georgetown University Press; 2000.

31. Comité de los Derechos del Niño, Naciones Unidas, Observación General Número 9, Derechos de los niños con discapacidad, 2006 [cited 2010 AUg 22], Available from: URL: http://www.iom.int/jahia/webdav/shared/shared/mainsite/ policy_and_research/un/63/es/A_63_41_ES.pdf

Recibido: 5 de julio de 2013

Aceptado: 30 de octubre de 2013 